\title{
Uber zwei bemerkenswerte russische pollenanalytische Arbeiten
}

\author{
Von Burkhard Frenzel, Freising-Weihenstephan
}

Mit 2 Abbildungen im Text

Der weite Bereich, der sich in Eurasien und Nordamerika während der pleistozänen Eiszeiten südlich der Inlandeismassen erstreckte und in dem die Vegetation unter dem Einfluß der damaligen Klimaungunst starke Veränderungen gegenüber dem heutigen und den interglazialen Verhältnissen erlitten hatte, liefert uns in zunehmendem Maße wichtige Erkenntnisse über die so verschiedenartigen und faszinierenden Probleme des Pleistozäns. Denn in diesem ehemals extraglazialen Gebiet haben sich an geeigneten Stellen größere Schichtpakete in ungestörter Lagerung erhalten können, die es uns infolge ihres Fossiliengehaltes gestatten, Einblicke in den Ablauf größerer Abschnitte des Pleistozäns zu gewinnen. In dieser Beziehung verdienen die Untersuchungen von I. A. DaniLova (1959) und M. P. Gričuk (1959) besondere Aufmerksamkeit, da es einerseits Danilova gelang, in einem einzigen Profil lakustriner Sedimente die Vegetationsgeschichte Mittelrußlands seit Beginn des letzten Interglazials (Eem) in groben Zügen aufzudecken und da andererseits GRIčUk auf Grund sehr zahlreicher Pollenanalysen die Veränderungen des Pflanzenkleides Baikaliens seit der Grenze Pliozän/Pleistozän in ihren wichtigsten Etappen rekonstruieren konnte.

$110 \mathrm{~km}$ westlich Moskaus befindet sich im Quellgebiet der Severnaja Bodnja ein fossiles Seebecken $(100-125 \mathrm{~m}$ mal $300-320 \mathrm{~m}$ ), das in einen Moränenrücken eingesenkt ist. Das Becken wird gegenwärtig von einem Cyperaceae-Sphagnum-Moor eingenommen. In diesem Moor wurde die Bohrung XII-51 niedergebracht, die folgende Sedimente erschloß (vgl. auch Abb. 1; Danilova 1959).

1) $0,00-0,85 \mathrm{~m}$ Schwach zersetzter Sphagnumtorf, mit vielen schlecht zersetzten Wurzeln; hellbraun, nach unten dunkler.

2) $\quad 0,85-1,07 \mathrm{~m}$ Brauner, toniger Torf; mit Pflanzenresten.

3) 1,07- 1,75 m Grauer Ton mit gelblich-graublauen Flecken, an der Luft gelblich-olivgrün werdend; homogen, plastisch, feinkörnig.

4) 1,75-2,30 m Grauer Ton mit stahlblauen Flecken, an der Luft olivgrün werdend; homogen, feinkörnig, plastisch, dichter als Schicht 3.

5) 2,30-2,60 $\mathrm{m}$ Dunkelgrauer Ton, mit olivgrünen bis bräunlichen Flecken; dicht, mir leicht fauligem Geruch; staubig-sandig, im unteren Teil mit Tonen, die. reich an organischem Material sind.

6) 2,60-3,00 m Faulschlamm, bräunlich bis dunkelgrau, mit olivgrünen Flecken; plastisch, dicht, riechend.

7) 3,00-3,82 m Faulschlamm, graubraun, mit schwärzlichen Flecken; dicht, plastisch, riechend.

8) 3,82- 4,10 m Mineralisierter Torf, hellbraun, locker, riechend, mit unzersetzten Pflanzenresten. In 4,00 m Tiefe wurde ein Menyanthes-Same gefunden.

9) 4,10- 4,67 m Faulschlamm, graubraun mit olivgrünen Flecken; plastisch, feinkörnig, dicht.

10) 4,67- 5,25 m Torfiger Ton, bräunlich, mit Pflanzenresten, riechend.

11) 5,25- 8,00 m Mineralisierter Torf, dunkelbraun, locker, mit Pflanzenresten. In $6,25 \mathrm{~m}$, $7,25 \mathrm{~m}$ und $7,50 \mathrm{~m}$ Tiefe Holzstückchen von Betula.

12) 8,00-9,62 m Bräunlich-schwarzer Torf, dicht, riechend, mit Resten von Betula-Holz. Proben zwischen 8,25 und $8,75 \mathrm{~m}$ Tiefe bestehen fast ausnahmslos aus Holz; es wurde offenbar ein Baumstamm erbohrt.

13) 9,62-10,00 m Brauner Faulschlamm, sehr dicht.

14) 10,00-10,50 m Faulschlammiger Ton, bräunlich bis olivgrün, sehr dicht; im unteren Teil mit Einschlüssen feinkörnigen, hellgrauen bis dunkelgrauen Sandes.

15) 10,50-10,87 m Hellgrauer Sand mit bläulichen Flecken; fein- bis grobkörnig; wasserführend; geht allmählich in liegende Schicht über.

16) 10,87-11,50 m Grauer Lehm mit bläulichen Flecken; dicht, mit kleinen Kalkstïcken. 

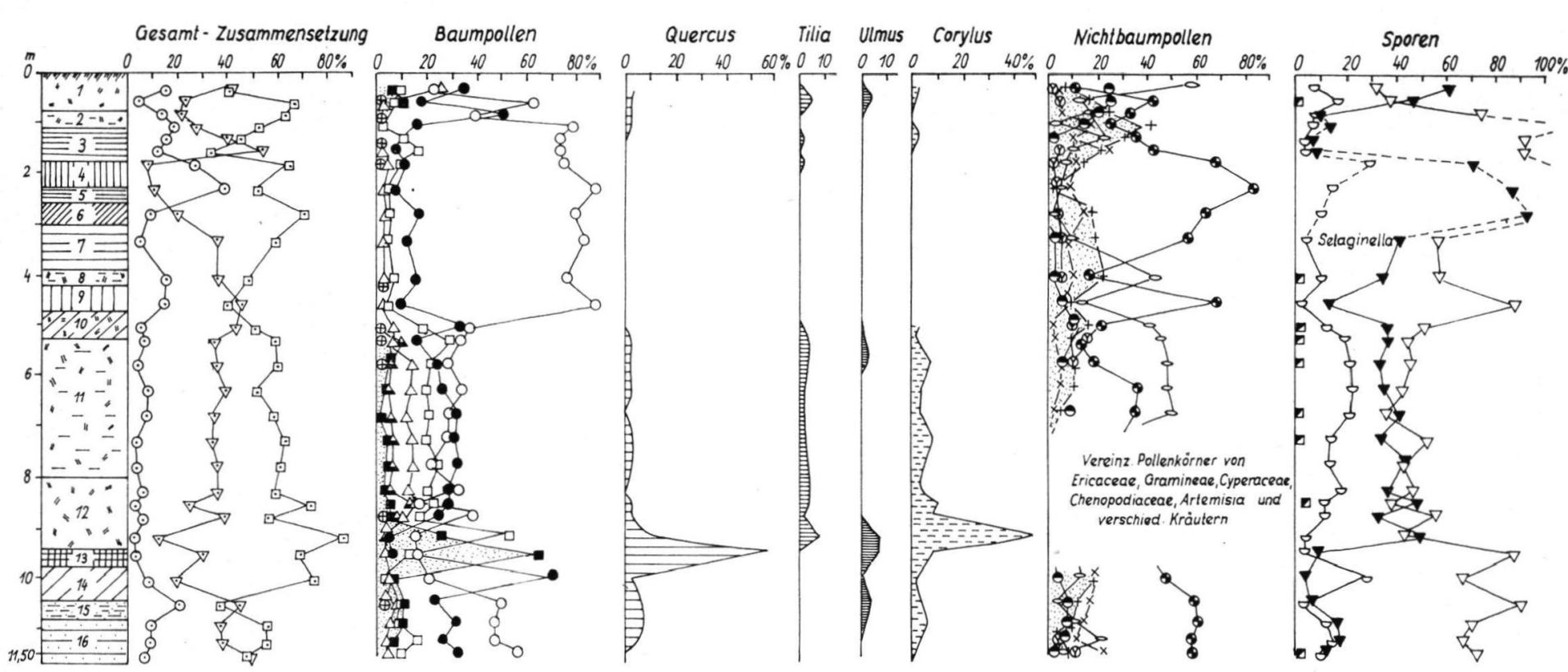

Abb. 1. Pollendiagramm lakustriner Sedimente im Quellgebiet der Severnaja Bodnja (Bohrung XII-51), 110 km westlich Moskaus. Aus: DanILova 1959. Die im Sedimentprofil stehenden Zahlen entsprechen denjenigen der Liste auf S. 211.
๑ Baumpollen (BP)
$\odot$ Nichtbaumpollen (NBP)
$\nabla$ Sporen
Baumpollen:
- Betula
- Pinus
$\triangle$ Picea
$\oplus$ Salix

- Alnus

$\triangle$ Carpinus

- Eichenmischwald (EMW) Nichtbaumpollen:

- verschiedene Kräuter

- Cyperaceae

- Gramineae

- Ericaceae

+ Artemisia

$\times$ Chenopodiaceae Sporen:

$\nabla$ Bryales

- Sphagnales

- Filices

- Lycopodiaceae 
Die pollenanalytische Bearbeitung des Bohrkerns ergab das in Abb. 1 dargestellte Bild. Die Schichten zwischen 11,50 und 10,00 m Tiefe werden durch ein anfängliches Birkenmaximum und einen darauffolgenden Pinus-Gipfel gekennzeichnet („1. Gruppe der Spektren", Danilova). Das Vorkommen von Quercus- und Ulmus-Pollen (zusammen maximal $11 \%$ ), sowie von Corylus und vereinzelter Carpinus in diesen Schichten führte DaniLova zu der Annahme, es handele sich hierbei um das Abbild eines Nadel-Laubmischwaldes des beginnenden Interglazials. Das erwähnte merkwürdige erste Quercus-, Ulmus- und Corylus-Maximum, das für letztinterglaziale Sedimente Mittelrußlands völlig ungewöhnlich ist, wie auch das sporadische Auftreten von Carpinus bei gleichzeitiger starker Beteiligung von Chenopodiaceae und Artemisia an der NBP-Flora lassen jedoch vermuten, daß hier sehr verschieden alte Pollen miteinander vermischt wurden, zumal da der erste EMWGipfel nicht in den lakustrinen Faulschlammen, sondern in Sanden und kalkskelettreichen Lehmen auftritt. Viel wahrscheinlicher als Danilovas Ansicht ist, daß die betreffenden Schichten die Pollenflora der ausklingenden Dneprovsk-Eiszeit (einschließlich MoskauStadium) enthalten, nämlich Hinweise auf eine unter einem trockenkalten Klima stehende Birken-Kiefern-, bzw. Kiefern-Birkenwaldsteppe (Artemisia, Chenopodiaceae, Ephedra!), die schließlich (ab 10,00 m) von edleren Laubwäldern verdrängt wurde.

Die optimale Entwicklung des EMW, mit den für das Eem-Interglazial in Mittelrußland so charakteristischen hohen Quercus-Werten und dem späten Auftreten von Corylus spiegeln die Schichten in 9,50 und 9,00 m Tiefe wider („2. Gruppe der Spektren“, DANILOVA). Ebenfalls für die Vegetationsfolge dieses Interglazials bezeichnend ist der auf das EMW-Maximum folgende Carpinus-Gipfel (8,50 m Tiefe), an den sich die Picea-Phase anschloß, während der Carpinus, Quercus, Tilia und Corylus jedoch noch nicht ganz verschwunden waren. DANilova nahm an, daß die Picea-Phase die Schichten von 8,75 bis 5,00 m Tiefe umfaßt („3. Gruppe der Spektren“, DANILOva). Es ist jedoch auffallend, daß mit dem Beginn der geschlossenen Artemisia- und Chenopodiaceae-Kurve in etwa $6,50 \mathrm{~m}$ Tiefe, d.h. ungefähr zu demselben Zeitpunkt, an dem die Vorherrschaft der BryalesSporen gegenüber den Sphagnales-Sporen einsetzte, die Kurven von Picea, Alnus, Quercus, Tilia und Corylus, in gewissem Sinne auch von Betula, einen spiegelbildlichen Verlauf zu demjenigen tieferer Horizonte nehmen und diese T'endenz mit Beginn der Bildung von Schicht 10 abrupt abbricht. Mit anderen Worten, es scheint so, als zeichne sich von etwa $6,50 \mathrm{~m}$ Tiefe der Beginn der Herrschaft eines trockenkalten Klimas ab, palynologisch jedoch verdeckt durch Pollen einer anspruchsvolleren Vegetation, die sich an sekundärer Lagerstätte befinden. Für die Richtigkeit dieser Deutung spricht, daß ein ähnlicher Kurvenverlauf in anderen Pollendiagrammen letztinterglazialer Sedimente meist nicht zu beobachten ist (vgl. Gričuk \& GričuK 1959, Čebotareva 1959) und daß SuKačEv und Mitarbeiter (1959) $10 \mathrm{~km}$ westlich Moskaus in offenbar gleichalten Sedimenten zusammen mit einer ähnlichen Pollenflora Makrofossilien von Salix berbacea, S. polaris, Betula cf. tortuosa, Alyssum sp. und der heute amphiatlantisch in Tundren bzw. im nördlichsten Bereich der Nadelwaldzone vorkommenden Draba cf. incana entdeckten. An die Stelle der interglazialen Wälder war damals somit die Strauchtundra getreten, in deren trockeneren Biotopen Kältesteppengesellschaften bereits Fuß gefaßt hatten.

Die Spektren von 4,75 bis etwa $1,00-1,50 \mathrm{~m}$ Tiefe rechnete DANILOva zu ihrer "4. Gruppe der Spektren“, die sie mit Recht als Abbild letzteiszeitlicher Verhältnisse ansah, ohne allerdings eine Gliederung dieses Abschnittes des jüngsten Pleistozäns durchzuführen. An Hand einiger weiterer pollenanalytischer Arbeiten, die in Mittelrußland in letzter Zeit durchgeführt worden sind, soll hier jedoch versucht werden, das interessante Profil weiter zu gliedern.

Im Wandel der Vegetation, der sich im Fossiliengehalt der Sedimente zwischen 5,00 und etwa $0,80 \mathrm{~m}$ Tiefe widerspiegelt, fallen zwei Phasen geringerer Bedeutung des Waldes und recht starker Beteiligung offener Vegetationstypen auf, von denen die ältere $(4,50$ bis 
4,00 m Tiefe) viel weniger scharf ausgeprägt war als die jüngere (2,50 bis 1,50 m Tiefe). Beide Phasen wurden durch einen Zeitraum beachtlicher Vorherrschaft der BP voneinander getrennt (3,50 bis 2,75 m Tiefe). Die Phasen stärkerer Beteiligung der NBP werden durch sehr hohe Werte der Kräuterpollen, der Bryales-Sporen, sowie durch Minima der Artemisia- und Chenopodiaceae-Pollen eingeleitet. Leider wissen wir nicht, welche Pflanzen die Kräuterpollen lieferten. Die starke Beteiligung der Bryales-Sporen, wie auch die Minima in den Kurven der xerophilen Artemisien und Chenopodiaceen deuten jedoch auf eine relativ gute Durchfeuchtung des Bodens zu Beginn der beiden NBP-reichen Phasen hin. Es ist bezeichnend, daß diese beiden Zeiten besserer Wasserversorgung jedoch von Episoden abgelöst wurden, in denen Chenopodiaceae und Artemisia eine außerordentlich große Rolle in der damaligen Vegetation spielten $(4,00-3,50 \mathrm{~m}$ Tiefe und 1,50 bis $0,80 \mathrm{~m}$ Tiefe) bei gleichzeitig beträchtlicher Verminderung der Bedeutung der BryalesSporen und der Kräuterpollen. Es ist sehr naheliegend, in diesem Vegetationswechsel das Abbild eines zweimaligen Überganges von einer Tundra- in eine Kältesteppenzeit, bzw. von der Fließerde- in die Lößzeit zu erblicken. Hierbei waren die erste Tundra- und Kältesteppenzeit sowohl was die Ausdehnung der offenen Pflanzengemeinschaften, als auch ihre Ausprägung anbetraf, weniger deutlich als die zweite. Beide NBP-reiche Phasen wie auch die zwischen ihnen gelegene BP-Phase wurden im Untersuchungsgebiet durch die Vorherrschaft von Betula gekennzeichnet, wobei jedoch unklar bleibt, welche Birkenart den Pollen während der einzelnen Abschnitte lieferte. Pinus spielte damals mit etwa 10\% der BP-Menge nur eine sehr untergeordnete Rolle, und Picea-Pollen traten sogar nur in wenigen Prozenten auf. Es ist allein nach diesem Profil fraglich, ob dem schwachen PiceaGipfel z.Zt. des hohen BP-Gipfels in $2,75 \mathrm{~m}$ Tiefe reale Bedeutung zukommt. Pollenanalytische Untersuchungen in benachbarten Gebieten Mittelrußlands lehren jedoch, daß die erwähnte BP-reiche Phase tatsächlich vielfach durch eine starke Zunahme der PiceaWerte gekennzeichnet war (Tabelle 1).

Hierbei ist wichtig, daß Picea auch während der älteren NBP-reichen Phase in allen betrachteten Gebieten prozentbildend vertreten war, obwohl diese Zeit durch heftige Solifluktionserscheinungen und eine Vergrößerung des Areals der ewigen Gefrornis bis

Tabelle 1

Prozentwerte der Pollen einiger wichtiger Pflanzen in der letzteiszeitlichen BP-Phase Mittelruß!ands

\begin{tabular}{|c|c|c|c|c|c|}
\hline \multirow[t]{2}{*}{ Pflanzen } & \multicolumn{5}{|c|}{ Lokalität } \\
\hline & $\begin{array}{c}110 \mathrm{~km} \\
\text { westl. Moskau } \\
\text { 1) }\end{array}$ & $\begin{array}{c}10 \mathrm{~km} \\
\text { westl. Moskau } \\
\text { 2) }\end{array}$ & $\begin{array}{l}\text { Unt.-u.Mittel- } \\
\text { lauf der Oka } \\
3)\end{array}$ & $\begin{array}{l}\text { P'joss a. d. } \\
\text { Wolga } \\
4)\end{array}$ & $\begin{array}{c}\text { Unterlauf } \\
\text { d. Kama } \\
5)\end{array}$ \\
\hline $\mathrm{BP}$ & $60-70$ & 64 & gleich bis & $40-60$ & $30-70$ \\
\hline NBP & $5-10$ & 36 & doppelt NBP & $15-40$ & $10-40$ \\
\hline Betula & ca. 80 & ca. 20 & $30-80$ & $70-80$ & $35-50$ \\
\hline Pinus & ca. 15 & ca. 40 & $30-70$ & $15-20$ & $25-46$ \\
\hline Picea & ca. 2 & bis 43 & $5-10(-30)$ & $0-20$ & $10-27$ \\
\hline Alnus & ca. 2 & - & $5-15$ & bis 3 & $4-17$ \\
\hline Kräuter & $50-60$ & Ephedra! & $30-70$ & $40-50$ & $15-55$ \\
\hline Artemisia & 15 & sehr viel & $10-50$ & $10-30$ & $0-2$ \\
\hline Chenopodiaceae & 10 & & $1-20$ & $10-15$ & $0-16$ \\
\hline
\end{tabular}

1) Danilova 1959; 2) Sukačev und Mitarbeiter 1959; 3) Aseev 1959; 4) Gričuk \& Gričuk 1959;

5) Moskvitin 1958. 
an das Nordufer des Kaspi-Sees ausgezeichnet war (u. a. Moskvitin 1958; weitere Literatur bei FRENZEL 1960a). In der BP-reichen Phase dehnten sich aber die Fichtenwälder beträchtlich aus, allerdings erreichten sie nicht mehr das heute in der Laubwaldzone gelegene Brjansk (VeličKo 1957). Diese Wälder, in denen die sibirische Picea obovata bis nach Moskau (SUKAčEv und Mitarbeiter 1959) vordrang, vermochten jedoch selbst damals nie, Mittelrußland geschlossen zu ïberziehen, sondern die trockenen Standorte wurden weiterhin von Artemisia- und Chenopodiaceae-Steppen eingenommen. Wenngleich somit das Klima in der BP-reichen Phase eine Ausdehnung der Wälder ermöglichte, so blieb der Klimacharakter doch trocken-kontinental. Erst die zweite NBP-reiche Phase, die die BPPhase ablöste, führte zu der fast vollständigen Vernichtung der Fichtenwälder (Pljoss, Kama, Moskau, Unter- und Mittellauf der Oka), ein weiteres Zeichen dafür, daß die zweite NBP-reiche Phase wesentlich einschneidender als die erste war. Auch in der zweiten NBP-reichen Phase waren Solifluktionsvorgänge weit verbreitet (FrENZEL 1960a). Diese zweite Tundren- und Kältesteppenzeit in Abb. 1 wurde durch eine letzte Phase etwas stärkerer Bewaldung und einen geringen Picea-Vorstoß (1,75 m Tiefe) gegliedert und mündete schließlich in die postglaziale Waldgeschichte ein, deren Beginn in Profil XII-51 etwas oberhalb $0,80 \mathrm{~m}$ Tiefe erschlossen wurde und die Danilova an Hand von Material einer zweiten Bohrung, die an derselben Lokalität niedergebracht wurde, eingehender untersuchte. Wie es für viele mittelrussische Pollendiagramme charakteristisch ist, wird auch in Abb. 1 der Übergang vom Pleistozän zum Holozän durch erste Tilia- und CorylusGipfel gekennzeichnet (Horizont der „Unteren Fichte"). Wahrscheinlich handelt es sich jedoch bei ihnen, ähnlich wie bei den entsprechenden ersten Gipfeln der edlen Laubhölzer an der Basis des Profils, um Pollen an sekundärer Lagerstätte, da die hohe Beteiligung der NBP an der gesamten Sporomorphenmenge der entsprechenden Horizonte, wie auch der starke Anteil von Artemisia- und Chenopodiaceae-Pollen nur schwer mit einem gleichzeitigen Tilia- und Cory'lus-Vorstoß zu vereinbaren ist $\left.{ }^{\top}\right)$. Es zeigt sich also, daß es DANILOVA tatsächlich gelungen ist, in einem einzigen Profil lakustriner Sedimente die wesentlichsten Etappen des Jungpleistozäns und des Holozäns vom Ende der vorletzten Eiszeit bis in die Gegenwart zu erschließen. Besondere Beachtung hierbei verdient die von einer schwächeren älteren und einer stärkeren jüngeren NBP-reichen Phase eingeschlossene BPreiche Phase, die durch Ausbreitung der Fichtengehölze in Mittelrußland gekennzeichnet war. In Abb. 1 verschleiert die Betulı-Kurve wahrscheinlich manche wichtigen Züge der Vegetationsentwicklung, da die einzelnen Betula-Arten ja nicht sicher palynologisch voneinander geschieden werden können. Trotzdem lassen der Kurvenverlauf in Abb. 1 wie auch die übrigen erwähnten pollenanalytischen Untersuchungen Mittelrußlands so viele Gemeinsamkeiten mit den von EmiLIANI $(1955,1956,1958)$ konstruierten Temperaturkurven, sowie mit den u. a. von Woldstedt (1958) und Gross (1958) durchgeführten Gliederungsversuchen der letzten Eiszeit erkennen, daß ich die von den beiden Tundrenund Kältesteppenzeiten begrenzte Phase der Fichtenhaine innerhalb kühler Steppen als Abbild des „Göttweiger" Interstadials, die zweite Tundren- und Kältesteppenzeit als das Kältemaximum der letzten Eiszeit (Hauptwürm bzw. kältester Teil von Mittelwürm) und die erste Tundren- und Kältesteppenzeit als Ausdruck der während des Früh- oder Altwürms herrschenden Klimaverhältnisse ansehen möchte.

Während der pleistozänen Eiszeiten waren in weiten Gebieten Nord-Eurasiens Tundren- und Kältesteppen, Strauchtundren und Mischformationen aus Waldtundren und Waldsteppen an die Stelle der verschiedenen Waldtypen der heutigen Taigazone getreten (für die letzte Eiszeit vgl. Frenzel \& Troll 1952; Frenzel 1960 b). Nur an sehr wenigen geschützten Lokalitäten vermochten kleine Waldrefugien die ungünstigen eiszeitlichen

1) Zur Problematik des Horizonts der "Unteren Fichte“ vgl. Frenzel 1960 b. 
Klimaverhältnisse zu überdauern. Das einzige große Waldgebiet stellte während der letzten Eiszeit das mittelsibirische Bergland dar, das jedoch während der ungünstigsten Klimaperioden durch einen Steppengürtel von dem stark verkleinerten mandschurischen Waldgebiet getrennt war (FRENZEL 1960 b). Es ist nun von hohem Interesse, auf Grund der Arbeiten von GRIčUK (1959) und seinen Mitarbeitern erste Einblicke in die pleistozäne Geschichte dieses mittelsibirischen Waldlandes, und zwar in der weiteren Umgebung von Irkutsk, zu gewinnen (Abb. 2). Es muß jedoch hervorgehoben werden, daß die genannten Autoren die Flußterrassen, aus denen die Proben entnommen wurden, bedauerlicherweise weder zu den zahlreichen Vereisungsspuren der Sajane, noch zu den Resten der in diesem Gebiet so verbreiteten paläolithischen Stationen in Beziehung gesetzt haben, sondern den Wandel der Vegetation nur aus dem Verlauf der Pollenkurven und der Höhenlage der Terrassen erschlossen, so daß Abb. 2 mit manchen Unsicherheiten behaftet ist.

Den Übergang von den bei Alga im Barguzintal (östlich des Baikalsees) beobachteten Vegetationsverhältnissen des jüngsten Tertiärs (vgl. die beiden untersten Diagrammstücke in Abb. 2) zu den pleistozänen Bedingungen stellte die Phase I dar, in deren Mitte sich mit dem Verschwinden von Tsuga, Ilex, Juglans und Pterocarya, sowie mit Kurventiefpunkten von Picea und der edleren Kiefern eine erste Kaltzeit abzeichnet, die im Bergland zur lokalen Ausbreitung von Artemisia-Chenopodiaceae-Ephedra-Steppen führte. Mit dieser Kaltzeit hatten die erwähnten Steppen zum ersten Male in nennenswertem Maße von Baikalien Besitz ergreifen können und wurden seither nie mehr vollständig verdrängt. Gričuk sieht die besprochene Kaltzeit als die erste pleistozäne Eiszeit an, die von einem ausgedehnten Interglazial (Phase II) gefolgt wurde, in dem anspruchsvollere Waldtypen aus Picea, Abies und Pinus Sekt. Strobus das Bild beherrschten. Das Areal

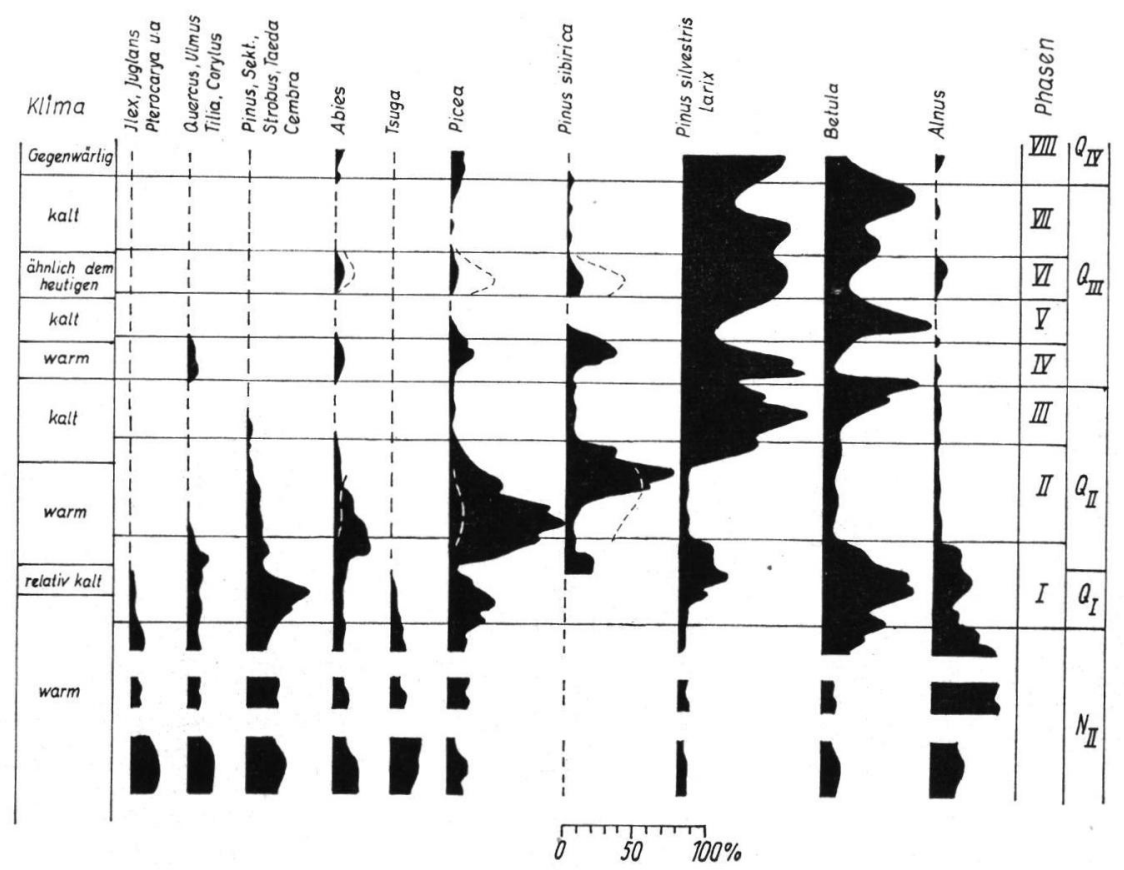

Abb. 2. Schematisiertes Pollendiagramm aus dem Flußgebiet der Angara, in der Umgebung von Irkutsk. Die Kurven stellen Mittelwerte zahlreicher, nur kürzere Abschnitte umfassender Pollendiagramme dar. Aus: Gričuk 1959.

Erklärung der Phasen im Text. N NII Neogen; QI: Altquartär; QII: Mittelquartär; QIII: Jungquartär; QIV: Holozön. - . - - Werte des nördlichen Angaragebietes. 
der Steppen war damals gegenüber den Verhältnissen der vorangegangenen Kaltzeit sehr stark eingeschränkt worden. Mit dem Übergang zur nächsten Eiszeit (Grenze der Phasen II/III und Phase III) stellten sich die für das heutige Waldkleid dieses Gebietes charakteristischen Pflanzengemeinschaften ein, zusammen mit ausgedehnten Steppen. Der Höhepunkt dieser Eiszeit führte jedoch zu einer sehr starken Verarmung der Pollenflora. Phase IV bezeichnet offenbar ein Interglazial, während dessen in der Umgebung von Irkutsk an mehreren Stellen Laubwälder aus Quercus, Ulmus, Tilia und Corylus gediehen. Diese Tatsache ist außerordentlich bemerkenswert, denn Tilia kommt heute nur in den nordwestlichen Vorbergen des Altai sowie im Ostteil Dahuriens und im Fernen Osten vor; ebenfalls in Dahurien erreichen Quercus mongolica und Corylus beterophylla als die am weitesten in den Kontinent von Osten her vorstoßenden Eichen- und Haselarten die Westgrenze ihrer Verbreitung, und lediglich Ulmus propinqua, sowie $U$. pumila dringen von Osten bis in das Flußgebiet der Selenga vor, erreichen jedoch Baikalien nicht mehr (Meusel 1943; Popov 1959). Die pollenanalytischen Befunde zeigen somit, daß Tilia nicht erst im Postglazial von Osten her in die nordwestlichen Vorberge des Altai eingewandert sein muß oder daß sie dort als Tertiärrelikt zu gelten habe (Literatur bei FRENZEL 1960 b), sondern daß noch im letzten Interglazial edle Laubwälder zwischen dem Altai und dem Fernen Osten vermittelten. Das erwähnte Interglazial wurde von einer weiteren Kaltzeit (Phase V) abgelöst, während der in Baikalien lediglich vereinzelte Pinus-, Larix- und Betula-Haine stockten. Eine ganz ähnliche, an Kältesteppen reiche Vegetation kennzeichnete die letzte Kaltzeit (Phase VII), die schließlich zu den postglazialen Waldtypen überleitete. Die beiden Kaltzeiten (Phasen V und VII) wurden durch eine Etappe stärkerer Bewaldung voneinander getrennt, während der die anspruchsvolleren Holzarten Picea, Abies und Pinus sibirica ihr Areal vergrößerten. Da die Pollenspektren dieser Zeit ungefähr denjenigen der rezenten Oberflächenproben gleichen, muß das damalige Klima dieses Raumes ungefähr dem heutigen entsprochen haben.

Die zeitliche Einordnung der von Gričuk herausgearbeiteten Phasen der Vegetationsgeschichte bereitet einige Schwierigkeiten, da, wie erwähnt, die Lagebeziehungen der einzelnen Fundhorizonte zu den glazigenen Sedimenten der Sajane sowie zu den paläolithischen Stationen und zu den Resten fossiler Großsäuger nicht berücksichtigt wurden. Daran, daß Phase VIII das Postglazial umfaßt, besteht kein Zweifel. Aus der Beschreibung der Fundorte geht außerdem hervor, daß die Proben, die zur Entdeckung der Phasen V, VI und VII führten, letzteiszeitlichen Terrassen entnommen wurden; denn die betreffenden Terrassen verzahnen sich nicht nur im Oberlauf der Flüsse mit Moränen zweier Talvergletscherungen, die jünger als eine Vergletscherung von Inlandeischarakter in den Sajanen sind, sondern sie enthalten auch jungpaläolithische Fundplätze (u. a. die Aurignacienstation Malta; Literatur vgl. Frenzel 1960a). Somit war die letzte Eiszeit in Baikalien in zwei Kältesteppenphasen mit Pinus-Larix-Betula-Hainen und in eine mittlere Waldphase, wahrscheinlich ein Interstadial ("Göttweiger“ Interstadial?), gegliedert. Das voraufgegangene Interglazial (Phase IV) dürfte jedoch dem Eem-Interglazial entsprechen, so daß GRIčuk wahrscheinlich mit Recht die Phasen IV und VII als das "Jungquartär" ansieht. Ob jedoch tatsächlich der oberste Teil der Phase I, sowie die Phasen II und III in das "Mittelquartär“ zu stellen sind und nur der untere Teil der Phase I das „Altquartär“ bezeichnet, wie Gričuk annimmt, erscheint fraglich. Denn einerseits deutet ein Vergleich der Spektren des unteren Teiles der Phase I mit den jungtertiären Spektren N II (unterstes Diagrammstïck) auf eine inzwischen eingetretene fühlbare Klimaverschlechterung vor Beginn der Phase I, und andererseits ist es möglich, daß in dem als Q II bezeichneten Abschnitt manche verschiedenalte Spektren der hochgelegenen und oft etwas undeutlichen Flußterrassen miteinander verknüpt wurden. Trotz dieser Bedenken läßt Abb. 2 jedoch die wesentlichen Etappen der Evolution der heutigen Vegetation dieses Raumes erkennen, und es wird deutlich, daß der heutige Taigatyp des Süd- 
teiles Mittelsibiriens sehr junger Entstehung ist und daß auch in dem mittelsibirischen Waldland die Einflüsse des mehrmaligen pleistozänen Klimawandels deutliche Spuren hinterlassen haben.

\section{Literaturverzeichnis}

Aseev, A. A.: Paläogeographie des Tales der mittleren und unteren Oka im Quartär. - Moskau 1959.

Čebotareva, N. S.: Stratigraphie der quartären Sedimente im Zentrum des Russischen Flachlandes. - In: Markov, K. K., \& A. I. Popov: Das Eiszeitalter im Europäischen Teil der UdSSR und in Sibirien, S. 116-147, Moskau 1959.

Danilova, I. A.: Quartäre Sedimente und das Relief in der Umgebung der geographischen Station „Krasnovidovo“ der Moskauer Staats-Universität. - Ibid., S. 64-115, Moskau 1959.

Emiliani, C.: Pleistocene temperature variations in the Mediterranean. - Quaternaria 2, S. 87-98, Rom 1955. - - Note on absolute chronology of human evolution. - Science 123, S. 924 bis 926, 1956. - - Paleotemperature analysis of core 280 and pleistocene correlations. Journ. of Geol. 66, S. 264-275, Chicago 1958.

Frenzel, B.: Die Vegetations- und Landschaftszonen Nord-Eurasiens während der letzten Eiszeit und während der postglazialen Wärmezeit. I. Teil: Allgemeine Grundlagen. - Akad. d. Wiss. u. d. Lit., Abh. d. math.-nat. Kl. Jahrg. 1959, Nr. 13, Mainz 1960 [1960 a]. - Die Vegetations- und Landschaftszonen Nord-Eurasiens während der letzten Eiszeit und während der postglazialen Wärmezeit. II. Teil: Rekonstruktionsversuch der letzteiszeitlichen und wärmezeitlichen Vegetation Nord-Eurasiens. - Ibid., Jahrg. 1960, Nr. 6, Mainz 1960 [1960 b].

Frenzel, B. \& C. Troll: Die Vegetationszonen des nördlichen Eurasiens während der letzten Eiszeit. - E. u. G. 2, S. 154-167, 1952.

GričuK, M. P.: Ergebnisse einer paläobotanischen Erforschung quartärer Sedimente des Angaragebietes. - In: Markov, K. K., \& A. I. Popov: Das Eiszeitalter im Europäischen Teil der UdSSR und in Sibirien, S. 442-497, Moskau 1959.

Gričuk, V. P., \& M. P. Gričuk: Altlakustrine Sedimente im Gebiet von Pljoss. - Ibid., S. 39-63, Moskau 1959.

Gross, H.: Die bisherigen Ergebnisse von C14-Messungen und paläontologischen Untersuchungen für die Gliederung und Chronologie des Jungpleistozäns in Mitteleuropa und den Nachbargebieten. - E. u. G. 9, S. 155-187, 1958.

Meusel, H.: Vergleichende Arealkunde. Bd. 2, Karte 43a, 1943.

Moskvitin, A. I.: Quartäre Sedimente und die Entstehungsgeschichte des Mittellaufes des Wolgatales. - Akad. Nauk SSSR, Trudy Geol. Inst., Vyp. 12, Moskau 1958.

Popov, M. G.: Flora Srednej Sibiri. Bd. 2, Moskau 1959.

Sukačev, V. N., R. N. Gorlova, A. K. Nedoseeva und E. P. Metelceva: Über die Vegetation des Periglazialgebietes des Zentrums des Russischen Flachlandes. - Doklady Akademii Nauk SSSR 125, S. 393-396, 1959.

Veličко, A. A.: Zum Problem des geo!ogischen Alters und der stratigraphischen Bedeutung des oberen Paläolithikums. - Izvest. Akad. Nauk SSSR, Ser. geogr., 1957, Nr. 2, S. 33-51, 1957.

Woldstedt, P.: Eine neue Kurve der Würm-Eiszeit. - E. u. G. 9, S. 151-154, 1958.

Manuskr. eingeg. 21. 10. 1960.

Anschrift des Verf.: Priv.-Doz. Dr. Burkhard Frenzel, Freising-Weihenstephan, Botanisches Institut der Technischen Hochschule München. 